\title{
Comparative study on the analytical performance of three waveforms for the determination of several aminoglycoside antibiotics with high performance liquid chromatography using amperometric detection
}

\author{
Yaqi Cai ${ }^{a}$, Yu'e Cai ${ }^{b}$, Jun Cheng ${ }^{c}$, Shifen Mou ${ }^{a}, *$, Lu Yiqiang ${ }^{b}$ \\ a State Key Laboratory of Environmental Chemistry and Ecotoxicology, Research Center for Eco-Environmental Sciences, \\ Chinese Academy of Sciences, P.O. Box 2871, Beijing 100085, China \\ b Department of Chemistry, University of Science and Technology Beijing, Beijing 100083, China \\ c Dionex Corporation, Sunnyvale, CA 94088-3603, USA
}

Available online 9 February 2005

\begin{abstract}
A preliminary comparative study was carried out on the analytical performances of a new six-potential waveform and other two detection waveforms, triple-potential waveform and quadruple-potential waveform. The analytical performances compared included signal response, background noise, signal/noise ratio and signal stability. Compared with triple-potential waveform and quadruple-potential waveform, the new six-potential waveform had higher signal response, signal/noise ratio, and sensitivity. As for determination reproducibility, the six-potential waveform also exhibited a slightly better performance than the other two waveforms. Under the selected experimental conditions based on the six-potential waveform, there is a linear correlation between peak area and concentration over two to three orders of magnitude for nine aminoglycoside antibiotics with a correlation coefficients better than 0.998 and the detection limits measured as three times the peak height signal-to-noise ratio for the nine aminoglycoside antibiotics were in the range of $0.0198-0.889 \mu \mathrm{g} / \mathrm{mL}$. The proposed method had been used to analyze real gentamicin sulphate drug sample.
\end{abstract}

(C) 2005 Elsevier B.V. All rights reserved.

Keywords: Waveforms; Amperometric detection; Aminoglycoside antibiotics; High performance liquid chromatography

\section{Introduction}

The aminoglycosides are a large class of antibiotics that characteristically contain two or more aminosugar groups linked by glycosidic bonds to an aminocyclitol component in their molecule strictures. They are a class of broad spectrum water-soluble antibiotics that exhibit very effective activity against some gram-positive and many gram-negative bacteria. But many aminoglycoside antibiotics have a very narrow margin between their therapeutic and toxic dose, for example, a too high plasma level of some aminoglycoside antibiotics may cause serious adverse ototoxicity and nephrotoxocity, so simple, sensitive and accurate analytical methods of aminoglycoside antibiotics in various drugs

\footnotetext{
* Corresponding author. Tel.: +86 106284 9182; fax: +861062849239.

E-mail address: shifenm@mail.rcees.ac.cn (S. Mou).
}

and body fluids are in great demand in medical practice for dosing guide, toxicity preventing and efficacy ensuring; some minor impurities containing in commercial bulk pharmaceuticals and their formulations also can exert harmful effects to human being's healthy, so quality control and quality assurance on aminoglycoside antibiotics' production also entail sensitive and reliable analytical techniques; in addition, some aminoglycoside products are extensively used in stockbreeding, and therefore they can find a way to many foods, so it is necessary to have sensitive and reproducible methods that possess capacity of determining its residues in tissues of farm animals, milk and meat containing foods.

Of various analytical methods available today, enzyme immunoassay methods are simple and sensitive, but they are suffered from the interferences of other co-existing antibiotics, the active metabolites and degradants of aminoglycoside antibiotics [1-4]. The mass spectrometry based liquid 
chromatography methods are specific, sensitive and simple, but instruments involved in this method are usually expensive and not available in many laboratories $[5,6]$. As for the high performance liquid chromatography (HPLC) methods based on the UV detection, because of the lack of strong chromophores in the molecule strictures, pre- and post-column derivertizations become indispensable and poor derivertization yield and low stability of derivatives also are problems waiting to be resolved in many cases [7-10]. Moreover, many major and minor UV detectable ingredients in pharmaceutical intermediate and final products give rise to a lot of serious interferences to the detection of aminoglycoside antibiotics.

Pulsed amperometric detection (PAD) is a sensitive method for the direct detection of aminoglycoside antibiotics in high $\mathrm{pH}$ on gold electrodes following high performance liquid chromatography (HPLC) separation. In those early works done by Adams et al. [11-15], a triple-potential detection waveform was commonly used. In the triple-potential waveform, to clean the surface fouling of gold electrode from oxidized analytes and other possible contaminants, a positive cleaning potential $(0.75 \mathrm{~V})$ was adopted. But exerting such a high positive cleaning potential as $0.75 \mathrm{~V}$ on a gold working electrode could cause an excessive gold oxide formation. The dissolution of gold oxide from the electrode surface results in a slow recession of gold electrodes. Consequently, the detector response decreases and the reproducibility becomes poorer and poorer over time. To overcome these drawbacks, Ding et al. [16] and Cai et al. [17] proposed some new quadruple-potential waveforms for the determination of neomycin and netilmicin. In the quadruple-waveform, a negative cleaning potential $(-2.0 \mathrm{~V})$ was selected to clean off gold surface. The new quadruple-potential waveform can improve the long-term reproducibility and alleviate the recession of the gold working electrode. But we noticed that with regard to many analytical performances such as detection sensitivity, the modified quadruple-waveform is only slightly better than the triple-potential waveform. Considering that the oxidation of the gold at the electrode surface definitely occur and the current from this process always contributes to the background, baseline noise and then results in poor sensitivity and reproducibility, to enhance signal and reduce baseline noise, Clarke et al. [18] introduced a six-potential waveform based integrated amperometry to detect amino acids. Unlike pulsed amperometry, in integrated amperometry, the potential during current sampling is not held constant but it is scanned between a high and low value. In this process, at the higher potential, the oxidation of analytes and gold at the surface of the electrode happens simultaneously, but at the lower potential, the gold oxide formed before is reduced. Because of the reversibility of gold oxidation and reduction process at the electrode surface and the irreversibility of analyte oxidation process, the contribution of gold oxidation to the signal is dramatically offset. The net signal only from the analyte is obtained by integrating the current during the whole high/low potential cycle. By using this six-potential waveform based integrated amperometry, in some extents, many analytical performances such as sensitivity and reproducibility can be improved greatly. To the best of our knowledge, the new waveform has not been used to the determination of aminoglycoside antibiotics. In view of the fact that the aminoglycoside antibiotics, carbohydrates and amino acids are all hydroxyl- and amino-containing compounds, it can be deduced that six-potential waveform based integrated amperometry maybe help improve the analytical performances of HPLC using amperometric detection for the determination of aminoglycoside antibiotics.

In this article, we introduced a modified six-potential waveform based integrated amperometry for the detection of several common aminoglycoside antibiotics, and made a comprehensive comparative study on the analytical performances of three waveforms, triple-, quadruple- and six-potential waveform, for the determination of several aminoglycoside antibiotics with high performance liquid chromatography using amperometric detection. The experimental results show that compared with triple-potential waveform and quadruple-potential waveform, the new six-potential waveform indeed exhibits a higher signal/noise ratio, and therefore enhances the sensitivity. In respect to other analytical performances such as reproducibility, the six-potential waveform also provides a slightly better performance than the other two waveforms.

\section{Experimental}

\subsection{Reagents}

To prepare the standard solutions and the mobile phase, $18 \mathrm{M} \Omega \mathrm{cm}$ purified water produced with a laboratory water purification system (Barnstead, IA, USA) was used throughout the experiments. Gentamicin sulfate CRS mixture (26.5\% $\mathrm{C}_{1}, 33.1 \% \mathrm{C}_{1 \mathrm{a}}, 26.0 \% \mathrm{C}_{2}$ and $14.6 \% \mathrm{C}_{2 \mathrm{a}}$ ), netilmicin sulfate CRS, sisomicin sulfate CRS, $1-N$-ethylgaramine sulfate CRS, neomycin B sulfate CRS (Council of Europe European Pharmacopoeia BP 907-F67029 Strasbourg Cedex 1) and neamine was purchased from Pierrel (Capua, Italy). Sodium 1-ocyanesulfonate was obtained from Acros Chimica (Geel, Belgium). Analytical reagent tetrahydrofuran (THF), guarantee grade reagent sodium sulfate anhydrous, sodium hydroxide, $85 \%$ phosphoric acid and potassium dihydrogen phosphate were purchased from Beijing Chemicals Corporation (Beijing, China). Gentamicin samples were provided by Yandou (Yandou, Shandong, China).

\subsection{Apparatus}

A ICS2500 ion chromatograph (Dionex, Sunnyvale, USA) was used throughout all experiments, and it consisted of a GP50 gradient pump with a built-in eluent degasser, an AS50 autosampler, a LC30 thermal compartment, a pneumatic device for post-column addition of sodium hydroxide and an ED50A electrochemical detector equipped 
with a thin-film type of three-electrode amperometric cell. The three-electrode amperometric cell included a working electrode made from a $3.0 \mathrm{~mm}$ diameter gold rod force-fit into a Kel-F plastic block, a $\mathrm{Ag} / \mathrm{AgCl}$ reference electrode $(3 \mathrm{M} \mathrm{KCl})$ and a stainless steel counter electrode. The detection thin-film channel of the cell was defined with the gasket dimensions (thickness $\times$ fluid channel width $\times$ fluid channel length): $0.140 \mathrm{~mm} \times 3.5 \mathrm{~mm} \times 14 \mathrm{~mm}$. PeakNet 6.1 software (Dionex, Sunnyvale, USA) was used for the chromatographic system control, data acquisition and analysis.

\subsection{Chromatography and PAD measurement}

The separations of aminoglycoside antibiotics were achieved by ion-pairing reverse phase chromatography performed on a poly(styrene-divinylbenzene) PLRP-S column $(250 \mathrm{~mm} \times 4.6 \mathrm{~mm}, 1000 \AA, 8 \mu \mathrm{m}$, Polymer Laboratories, Shropshire, UK) installed inside the LC30 thermal compartment for keeping a constant temperature.

The buffer solution ( $\mathrm{pH}$ 3.0) used for the preparation of mobile phase was prepared by mixing appropriate amount of $0.20 \mathrm{M}$ potassium dihydrogen phosphate and $0.20 \mathrm{M}$ phosphoric acid. The 50\% sodium hydroxide concentrated solution was prepared by dissolved suitable amount of solid sodium hydroxide into equal amounts of purified water. To precipitate as more amounts of sodium carbonate as possible before use, this solution was left undisturbed for at least $24 \mathrm{~h}$. Post-column adding reagent $0.50 \mathrm{M}$ sodium hydroxide was made using above-mentioned $50 \%(\mathrm{w} / \mathrm{w})$ sodium hydroxide aqueous dilution.

Because of different property, each aminoglycoside antibiotic exhibits different retention behavior, so each aminoglycoside antibiotic surely has its own optimal chromatographic condition. For convenience, we divided aminoglycoside antibiotics into three groups, the first one included gentamicin $\mathrm{C}_{1}, \mathrm{C}_{1 \mathrm{a}}, \mathrm{C}_{2}$ and $\mathrm{C}_{2 \mathrm{a}}$, the second one included netilmicin, sisomicin and $1-N$-ethylgaramine, and the last one included neomycin B and neamine. We adopted three different chromatographic conditions for every group respectively, these chromatographic conditions were very similar to those of European Pharmacopoeia method except some minor adjustments and they are shown in Table 1. In all experiments, the mobile phase flow rate was set at $1.0 \mathrm{~mL} / \mathrm{min}$. Post-column reagent $0.50 \mathrm{M}$ sodium hydroxide was added through a mixing Tee at a flow rate of $0.30 \mathrm{~mL} / \mathrm{min}$, then the sodium hydroxide and mobile phase were further mixed through a $375 \mu \mathrm{L}$ knitted Teflon coil prior to reach the PAD detection cell. The stock solutions of the aminoglycoside antibiotics with concentration of $1 \mathrm{mg} / \mathrm{mL}$ were prepared by dissolving suitable amount of their CRSs in their own mobile phases, all injected standard mixture solutions of the three groups of aminoglycoside antibiotics were made by diluting the corresponding aliquots of stock solutions with their own mobile phase. Twenty-microliter solution was injected into the chromatographic system for the determination. The mobile phases and post-column reagent $0.50 \mathrm{M}$ sodium hydroxide were all kept under nitrogen to prevent contamination from atmospheric carbon dioxide.

To compare the analytical performances of different waveforms for the detection of several aminoglycoside antibiotics, three detection potential waveforms used are presented in Fig. 1.

\section{Results and discussion}

\subsection{Chromatographic conditions}

By making some careful and minor adjustment to the European Pharmacopoeia method according to our own laboratory experimental conditions, we got relative optimized chromatographic conditions for our own chromatographic system and they are shown in Table 1. Under these conditions, we can complete one chromatographic process for the separation of five gentamicin components: $\mathrm{C}_{1 \mathrm{a}}, \mathrm{C}_{2 \mathrm{~b}}, \mathrm{C}_{2}, \mathrm{C}_{2 \mathrm{a}}$ and $\mathrm{C}_{1}$ in about 40 min with a satisfactory resolution that can meet the demand of European Pharmacopoeia method (peak-to-valley ratio $\left(\mathrm{C}_{2} / \mathrm{C}_{2 \mathrm{a}}\right)$ greater than 2$)$. As for the separation of ethylgaramine, sisomicin and netilmicin, one chromatographic process can be completed within about 9 min with a very good resolution that far exceed the demand of European Pharmacopoeia method (Resolution(ethylgaramine/sisomicin) greater than 2, Resolution(sisomicin/netilmicin) greater than $3)$. In the case of the separation of neamine and neomycin, one chromatographic run can be gone through in $13 \mathrm{~min}$ and good separation can be obtained. The chromatograms of the three systems are given in Figs. 2-4, respectively.

\subsection{Effect of waveform on the detection of nine aminoglycoside antibiotics}

Our main goal in this paper is making comparison of three different waveforms for their detection aspects of nine aminoglycoside antibiotics. The triple-potential waveform

Table 1

Chromatographic conditions of three groups of aminoglycoside antibiotics

\begin{tabular}{lccc}
\hline & Gentamicin mixture & Netilmicin, sisomicin and 1- $N$-ethylgaramine & Neomycin B and neamine \\
\hline $\mathrm{Na}_{2} \mathrm{SO}_{4}(\mathrm{~g} / \mathrm{L})$ & 60 & 38 & 70 \\
Sodium 1-ocyanesulfonate $(\mathrm{g} / \mathrm{L})$ & 1.75 & 0.4 & 1.4 \\
$\mathrm{pH} 3.0$ buffer $(\mathrm{mL} / \mathrm{L})$ & 50 & 50 & 50 \\
$\mathrm{THF}(\mathrm{mL} / \mathrm{L})$ & 8.0 & 13 & 5.0 \\
Column temperature $\left({ }^{\circ} \mathrm{C}\right)$ & 55 & 40 & 35 \\
\hline
\end{tabular}



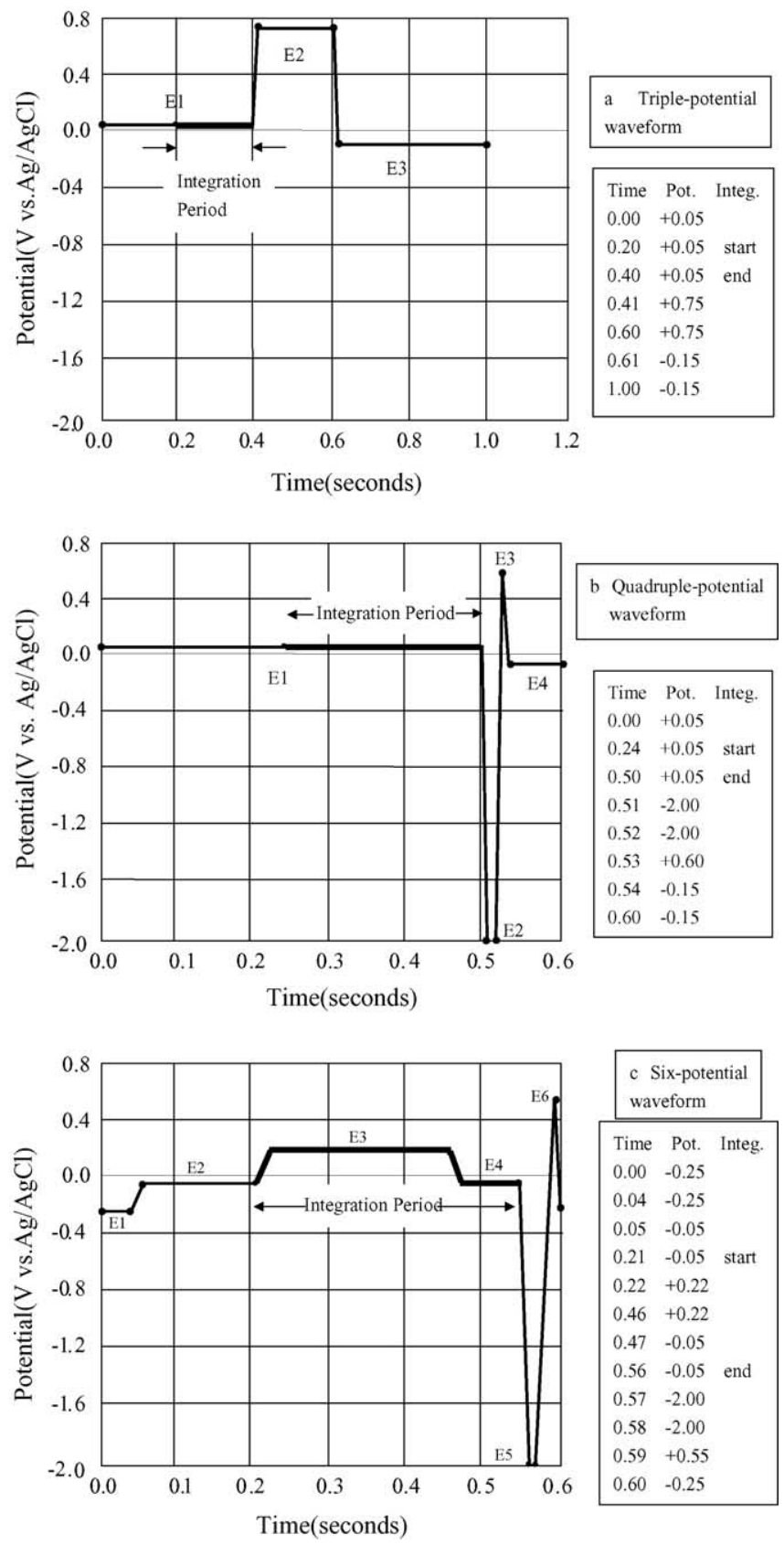

Fig. 1. Three waveforms for the amperometric detection of aminoglycoside antibiotics: (a) triple-potential waveform; (b) quadruple-potential waveform; (c) six-potential waveform.

we adopted was recommended by Adams et al. [11-15] and it is accepted by current European Pharmacopoeia method, this waveform is involved in the use of a relatively long period of high positive cleaning potential, which makes the surface of the gold working electrode recede gradually, the detection response decrease and the reproducibility become worse and worse over time. The quadruple-potential waveform in this paper was the same as we used before [17] and it is involved in the use of a negative cleaning potential instead of a relatively long period of high positive cleaning potential, which minimizes the problem of electrode recession and improves the sensitivity and reproducibility. The six-potential waveform chosen in this paper was derived from the waveform introduced by Clarke et al. [18] for the integrated pulsed amperometry detection of amino acids and amino sugars. After some preliminary optimization conducted by varying single potential while keeping the other parameters of waveforms constant, we achieved a fairly perfect six-potential waveform as shown in Fig. 1 for the detection of nine aminoglycoside antibiotics by integrated pulsed amperometry detection coupled with HPLC.

The waveform has a great influence on the signal response, background noise, signal/noise and signal stability, therefore it has great effect on the detection sensitivity and reproducibility. To select the most suitable waveform for our system, we make a careful investigation and comparison on the quantitative aspects of the three waveforms for the determination of nine aminoglycoside antibiotics for the first time. When conducting this comparative study, we make 14 successive injections for every experimental group analytes with the same concentration at each fixed waveform condition, then we can got every analyte's peak height, peak area, background noise and the relative standard deviation for peak height and peak area in every waveform conditions, then we can make a preliminary comparison between three waveforms for their quantitative detection performance and find out the best one. The experimental results we got are shown in Table 2, Figs. 2-4. From these results, we can find that as for the peak height and the peak area, the tripleand quadruple-potential waveform exhibit the roughly same analytical performance, but because of the slightly smaller background noise of the quadruple-potential waveform, the quadruple-potential waveform provides a slightly better signal/noise than the triple-potential waveform in the most cases. Compared with the triple- and quadruple-potential waveform, six-potential waveform has a very different situation. By adopting six-potential waveform, we can get about four to eight times signal response as high as those of the triple- and quadruple-potential waveform. In respect to the background noise, six-potential waveform provides a noise about twice as high as those of the triple- and quadruplepotential waveform in the most cases except for the detection of neamine and neomycin. From the above-mentioned experimental phenomenon, we notice that the introduction of the six-potential waveform simultaneously exert a positive and negative effect on the detection of the nine aminoglycoside antibiotics, it can not only increase the signal response, but also increase the background noise. Although higher background noise may cancel out parts of the signal response in some extent, because the increase of the signal response is much more remarkable than that of the background noise, the net outcome of signal/noise ratio using six-potential waveform is still much higher than those of the system using triple- and quadruple-potential waveform. From the Table 2, it is found that the signal/noise ratio of the system using six-potential waveform is two to four times as high as those 


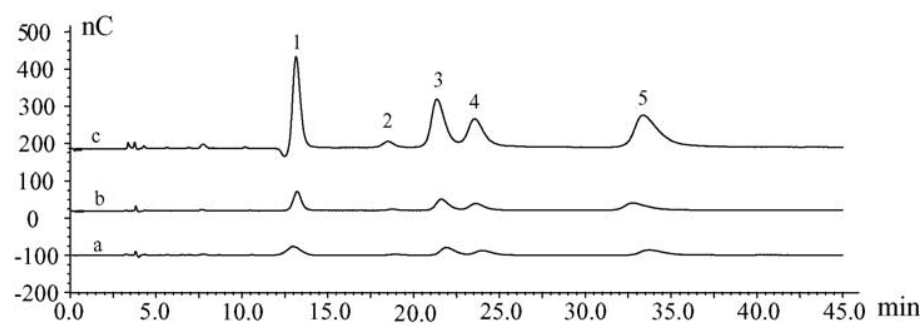

Fig. 2. Chromatograms of a standard mixture of gentamicin $\mathrm{C}_{1}, \mathrm{C}_{1 \mathrm{a}}, \mathrm{C}_{2}$ and $\mathrm{C}_{2 \mathrm{a}}$ obtained with chromatographic condition shown in Table 1. Injection volume is $20 \mu \mathrm{L}$; Concentrations of gentamicin $\mathrm{C}_{1}, \mathrm{C}_{1 \mathrm{a}}, \mathrm{C}_{2}$ and $\mathrm{C}_{2 \mathrm{a}}$ are $133,166,130$ and $73.0 \mu \mathrm{g} / \mathrm{mL}$, respectively; detection waveform: a, triple-potential waveform; b, quadruple-potential waveform; c, six-potential waveform; peak 1: gentamicin $\mathrm{C}_{1 \mathrm{a}}$; peak 2: gentamicin $\mathrm{C}_{2 \mathrm{~b}}$; peak 3: gentamicin $\mathrm{C}_{2}$; peak 4: gentamicin $\mathrm{C}_{2 \mathrm{a}}$; peak 5: gentamicin $\mathrm{C}_{1}$.

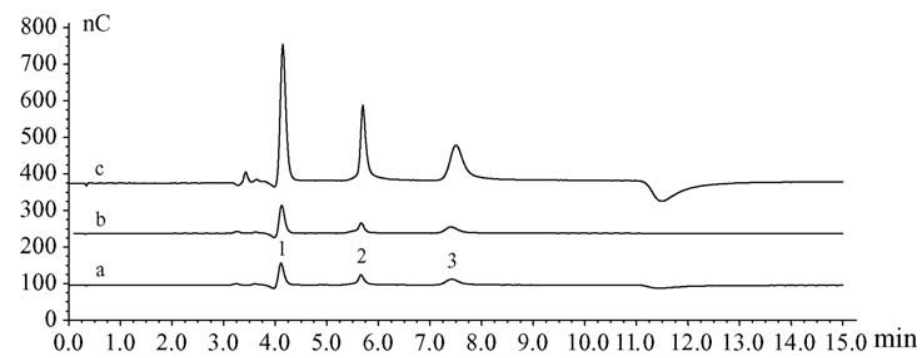

Fig. 3. Chromatograms of a standard mixture of ethylgaramine, sisomicin and netilmicin obtained with chromatographic condition shown in Table 1. Injection volume is $20 \mu \mathrm{L}$; analyte concentration is $10 \mu \mathrm{g} / \mathrm{mL}$; detection waveform: a, triple-potential waveform; b, quadruple-potential waveform; c, six-potential waveform; peak 1: ethylgaramine; peak 2: sisomicin; peak 3: netilmicin.

of the system using triple- and quadruple-potential waveform in most cases. Judging from above experimental results, we can come to a preliminary conclusion that six-potential waveform exhibits higher detection sensitivity than tripleand quadruple-potential waveform.

In respect to the signal stability, from the Table 2, although we notice that the RSD for the peak height in the system using six-potential waveform is slightly smaller than those of the system using triple- and quadruple-potential waveform, we cannot yet draw a clear conclusion on the matter which waveform exhibits better reproducibility. We think the reason about this is that the successive repetition injections are not many enough to make us obtain a regular pattern on this matter. In the case of six-potential waveform, RSD of peak heights of the nine analytes for 14 successive injections were no more than $7.7 \%$, it showed that the system had a good stability and reproducibility.

\subsection{Analytical performance of six-potential detection waveform}

Since the experimental results reveal to us that sixpotential waveform indeed had a more satisfactory detection performance for the nine aminoglycoside antibiotics, it was adopted in our rest experiments. Under this selected chromatographic and detection conditions, the analytical performances of the proposed method were examined and they are shown in Table 3. From this table, we can found that there is a linear correlation between peak area and concentration over two to three orders of magnitude for nine aminoglycoside antibiotics with a correlation coefficients better than 0.998 and the detection limits measured as three times the peak height signal-to-noise ratio for the nine aminoglycoside antibiotics were in the range of $0.0198-0.889 \mu \mathrm{g} / \mathrm{mL}$. The data in Table 3 indicated that the method we present here is fairly sensitive.

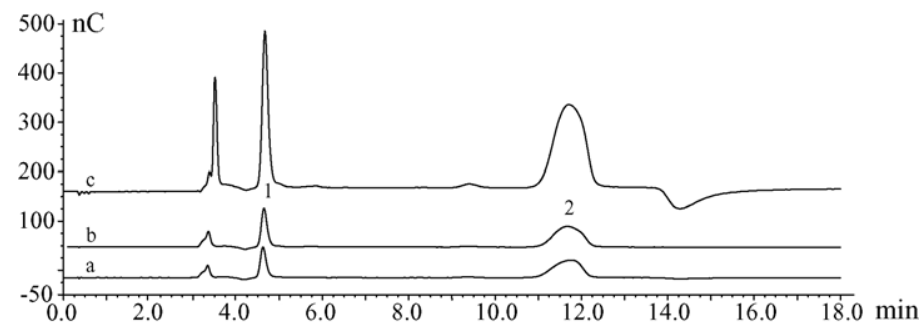

Fig. 4. Chromatograms of a standard mixture of neamine and neomycin obtained with chromatographic condition shown in Table 1 . Injection volume is $20 \mu \mathrm{L}$; analyte concentration is $10 \mu \mathrm{g} / \mathrm{mL}$; detection waveform: a, triple-potential waveform; b, quadruple-potential waveform; c, six-potential waveform; peak 1: neamine; peak 2: neomycin. 
Table 2

Comparison of three different waveforms on the peak height, RSD for peak height, noise and signal/noise ratio for nine aminoglycoside antibiotics

\begin{tabular}{|c|c|c|c|c|c|c|c|c|c|c|c|c|}
\hline & \multicolumn{4}{|c|}{ Triple-potential waveform } & \multicolumn{4}{|c|}{ Quadruple-potential waveform } & \multicolumn{4}{|c|}{ Six-potential waveform } \\
\hline & $H^{\mathrm{a}}(\mathrm{nC})$ & $\begin{array}{l}\text { RSD } \\
(H)^{\mathrm{b}}(\%)\end{array}$ & $N^{\mathrm{c}}(\mathrm{nC})$ & $\mathrm{S} / \mathrm{N}^{\mathrm{d}}$ & $H^{\mathrm{a}}(\mathrm{nC})$ & $\begin{array}{l}\text { RSD } \\
(H)^{\mathrm{b}}(\%)\end{array}$ & $N^{\mathrm{c}}(\mathrm{nC})$ & $\mathrm{S} / \mathrm{N}^{\mathrm{d}}$ & $H^{\mathrm{a}}(\mathrm{nC})$ & $\begin{array}{l}\text { RSD } \\
(H)^{\mathrm{b}}(\%)\end{array}$ & $N^{\mathrm{c}}(\mathrm{nC})$ & $\mathrm{S} / \mathrm{N}^{\mathrm{d}}$ \\
\hline \multicolumn{13}{|c|}{ Group I: gentamicin mixture $\mathrm{e}^{\mathrm{e}}$} \\
\hline $\mathrm{C}_{1}$ & 17.8 & 8.0 & 0.275 & 64.8 & 17.3 & 9.6 & 0.186 & 93.1 & 88.5 & 6.0 & 0.361 & 245.3 \\
\hline $\mathrm{C}_{1 \mathrm{a}}$ & 49.9 & 8.8 & 0.275 & 181.6 & 32.0 & 17.3 & 0.186 & 172.3 & 254.8 & 7.5 & 0.361 & 706.3 \\
\hline $\mathrm{C}_{2}$ & 28.0 & 8.3 & 0.275 & 102.1 & 25.0 & 10.0 & 0.186 & 134.7 & 128.9 & 7.4 & 0.361 & 357.3 \\
\hline $\mathrm{C}_{2 \mathrm{a}}$ & 17.0 & 8.0 & 0.275 & 69.7 & 15.0 & 9.6 & 0.186 & 80.9 & 74.1 & 7.7 & 0.361 & 205.4 \\
\hline \multicolumn{13}{|c|}{ Group II: ethylgaramine, sisomicin and netilmicin ${ }^{\mathrm{f}}$} \\
\hline Ethylgaramine & 63.6 & 2.6 & 0.238 & 267.1 & 77.6 & 3.5 & 0.240 & 323.8 & 373.1 & 1.8 & 0.460 & 811.5 \\
\hline Sisomicin & 26.2 & 6.0 & 0.238 & 110.0 & 27.0 & 3.8 & 0.240 & 112.8 & 201.5 & 1.7 & 0.460 & 438.3 \\
\hline Netilmicin & 15.1 & 3.6 & 0.238 & 63.8 & 17.1 & 1.2 & 0.240 & 71.2 & 96.0 & 2.8 & 0.460 & 208.7 \\
\hline \multicolumn{13}{|c|}{ Group III: neamine and neomycin ${ }^{\mathrm{g}}$} \\
\hline Neamine & 62.8 & 1.3 & 0.146 & 429.1 & 74.1 & 5.3 & 0.0985 & 752.1 & 313.9 & 1.8 & 0.354 & 888.0 \\
\hline Neomycin & 35.7 & 4.7 & 0.146 & 244.0 & 41.8 & 5.5 & 0.0985 & 424.8 & 172.7 & 2.7 & 0.354 & 488.6 \\
\hline
\end{tabular}

a Average peak height for 14 successive injections.

${ }^{\mathrm{b}}$ Relative standard deviation of peak height for 14 successive injections.

c Average noise for 14 successive injections.

${ }^{\mathrm{d}}$ Average signal/noise ratio for 14 successive injections.

e Concentrations of analytes injected are $133,166,130$ and $73.0 \mu \mathrm{g} / \mathrm{mL}$ for gentamicin $C_{1}, C_{1 a}, C_{2}$ and $C_{2 a}$, respectively.

${ }^{\mathrm{f}}$ Concentrations of ethylgaramine, sisomicin and netilmicin in injected samples all are $10 \mu \mathrm{g} / \mathrm{mL}$

g Concentrations of neamine and neomycin in injected samples all are $10 \mu \mathrm{g} / \mathrm{mL}$.

Table 3

Quantitative aspects of the six-potential waveform based system for the determination of nine aminoglycoside antibiotics

\begin{tabular}{|c|c|c|c|c|}
\hline & Linear range $(\mu \mathrm{g} / \mathrm{mL})$ & Calibration curve $^{\mathrm{a}}$ & $\begin{array}{l}\text { Correlation } \\
\text { coefficient }\end{array}$ & $\begin{array}{l}\text { Limit of detection } \\
(\text { signal/noise ratio }=3)(\mu \mathrm{g} / \mathrm{mL})\end{array}$ \\
\hline \multicolumn{5}{|c|}{ Group I: gentamicin mixture } \\
\hline $\mathrm{C}_{1}$ & $2.65-530$ & $A=2.6275 c-0.6111$ & 0.9999 & 0.266 \\
\hline $\mathrm{C}_{1 \mathrm{a}}$ & $3.31-662$ & $A=1.5760 c+13.561$ & 0.9982 & 0.889 \\
\hline $\mathrm{C}_{2}$ & $2.60-520$ & $A=1.9326 c+12.641$ & 0.9990 & 0.371 \\
\hline $\mathrm{C}_{2 \mathrm{a}}$ & $1.46-292$ & $A=2.7922 c+1.2216$ & 0.9999 & 0.392 \\
\hline \multicolumn{5}{|c|}{ Group II: ethylgaramine, sisomicin and netilmicin } \\
\hline Ethylgaramine & $0.25-50$ & $A=4.6171 c+0.8606$ & 0.9986 & 0.0263 \\
\hline Sisomicin & $0.50-50$ & $A=3.3767 c-1.5492$ & 0.9997 & 0.0765 \\
\hline Netilmicin & $0.50-50$ & $A=4.0341 c-3.4288$ & 0.9994 & 0.256 \\
\hline \multicolumn{5}{|c|}{ Group III: neamine and neomycin } \\
\hline Neamine & $1.0-50$ & $A=5.4435 c+1.5585$ & 0.9994 & 0.0198 \\
\hline Neomycin & $4.0-200$ & $A=3.4687 c+1.2409$ & 0.9993 & 0.0372 \\
\hline
\end{tabular}

${ }^{\mathrm{a}} A$ : peak area $(\mathrm{nC} \min ) ; c$ : concentration of analytes injected $(\mu \mathrm{g} / \mathrm{mL})$.

To examine the applicability of the proposed six-potential waveform based method, the proposed method had been applied to analyze real gentamicin sulphate drug sample. Because the present method is more sensitive than the triple-potential waveform based method adopted by European Pharmacopoeia, so the test solution we prepared was much more dilute than that recommended by European Pharmacopoeia $(50.0 \mathrm{mg}$ of gentamicin sulphate drug sample in $100 \mathrm{~mL}$ with the mobile phase), it was prepared by dissolved $25.0 \mathrm{mg}$ of gentamicin sulphate drug sample in $250 \mathrm{~mL}$ mobile phase solution. Following the European Pharmacopoeia 4.5 07/2003:0331 method, we completed the chromatographic analytical process. According to European Pharmacopoeia 4.5 07/2003:0331 method, the response factors for gentamicins $\mathrm{C}_{1 \mathrm{a}}, \mathrm{C}_{2}, \mathrm{C}_{2 \mathrm{a}}$ and $\mathrm{C}_{2 \mathrm{~b}}$ relative to $\mathrm{C}_{1}$ were all about one, then we can calculate every component's relative content by normalization and the results are shown

Table 4

Analytical results of gentamicin samples by normalization

\begin{tabular}{lc}
\hline & $\begin{array}{l}\text { Composition of } \\
\text { gentamicin samples }^{\mathrm{a}}(\%)\end{array}$ \\
\hline $\mathrm{C}_{1 \mathrm{a}}$ & $27.91 \pm 1.25$ \\
$\mathrm{C}_{2 \mathrm{~b}}$ & $2.28 \pm 0.14$ \\
$\mathrm{C}_{2}$ & $25.20 \pm 0.68$ \\
$\mathrm{C}_{2 \mathrm{a}}$ & $18.29 \pm 0.75$ \\
$\mathrm{C}_{1}$ & $26.33 \pm 1.83$ \\
\hline
\end{tabular}

${ }^{a}$ Mean and standard deviation for four determinations. 
in Table 4. We can find that our method has a good precision, which can guarantee us to obtain more reliable results.

\section{Conclusion}

In this paper, for the first time, a modified six-potential waveform based integrated amperometry coupled to the HPLC for the detection of several common aminoglycoside antibiotics was introduced, and a comprehensive comparative study on the analytical performances of this novel six-potential waveform and other two detection waveforms, triple-potential waveform and quadruple-potential waveform, was conducted. The experimental results show that compared with triple- and quadruple-potential waveform, the six-potential waveform provides higher signal response, signal/noise ratio, and therefore improves the sensitivity obviously. In respect to other analytical performances such as reproducibility, the six-potential waveform also exhibits a slightly better performance than the other two waveforms. The six-potential waveform might be applied in the field of aminoglycoside antibiotic analysis.

\section{Acknowledgements}

This work was jointly supported by National Basic Research Program of China (2003CB415001) and the National Natural Science Foundation of China (20475060).

\section{References}

[1] W. Haasnoot, P. Stouten, H. Cazemier, A. Lommen, J.F.M. Nouws, H.J. Keukens, Analyst 124 (1999) 301.

[2] A. Strasser, R. Dietrich, E. Usleber, E. Martlbauer, Anal. Chim. Acta 495 (2003) 11.

[3] W. Heering, E. Usleber, R. Dietrich, E. Martlbauer, Analyst 123 (1998) 2759.

[4] P. Schnappinger, E. Usleber, E. Martlbauer, G. Terplan, Food Agric. Immunol. 5 (1993) 67.

[5] M. van Bruijnsvoort, S.J.M. Ottink, K.M. Jonker, E. de Boer, J. Chromatogr. A 1058 (2004) 137.

[6] R. Oertela, V. Neumeisterb, W. Kircha, J. Chromatogr. A 1058 (2004) 197

[7] D.A. Stead, J. Chromatogr. A 747 (2000) 69.

[8] A. Posyniak, J. Zmudzki, J. Niedzielska, J. Chromatogr. A 914 (2001) 59.

[9] D.A. Stead, R.M.E. Richards, J. Chromatogr. B 693 (1997) 415.

[10] E. Roets, E. Adams, I.G. Muriithi, J. Hoogmartens, J. Chromatogr. A 696 (1995) 131.

[11] E. Adams, W. Roelants, R. De Paepe, E. Roets, J. Hoogmartens, J. Pharm. Biomed. Anal. 18 (1998) 689.

[12] E. Adams, G. Van Vaerenbergh, E. Roets, J. Hoogmartens, J. Chromatogr. A 819 (1998) 93.

[13] E. Adams, D. Puelings, M. Rafiee, E. Roets, J. Hoogmartens, J. Chromatogr. A 812 (1998) 151.

[14] E. Adams, R. Schepers, E. Roets, J. Hoogmartens, J. Chromatogr. A. 741 (1996) 233.

[15] J. Szunyog, E. Adams, E. Roets, J. Hoogmartens, J. Pharm. Biomed. Anal. 23 (2000) 891.

[16] Y. Ding, H. Yu, S. Mou, J. Chromatogr. A. 1039 (2004) 39.

[17] Y. Cai, J. Cheng, S. Mou, P. Jandik, Pharmacopeial Forum 30 (2004) 2.

[18] A.P. Clarke, P. Jandik, R.D. Rocklin, Y. Liu, N. Avdalovic, Anal. Chem. 71 (1999) 2774. 